\title{
Fast Mapping Of Biological Samples With Large Area EDS Detectors
}

\author{
C.L. Collins ${ }^{1}$, C. McCarthy ${ }^{1}$, N. Rowlands ${ }^{1}$, S.R. Burgess ${ }^{1}$ \\ ${ }^{1}$ Oxford Instruments Nanoanalysis, Halifax Road, High Wycombe, HP12 3SE, UK
}

Energy Dispersive Spectroscopy (EDS) has been used for many years to analyse the chemical composition of materials, including biological matter. However, the physical limitations of often being able to use only low beam currents, low accelerating voltages and only generating very low X-Ray count rates on a sample mainly comprised of light elements meant that until now, biological EDS has been somewhat limited in its practical application.

The introduction of new silicon drift detectors (SDDs) revolutionised many areas of SEM analysis with fast accurate EDS analysis and low data collection times [1], however, it is only very recently, with the introduction of new very large area SDD detectors $\left(80-150 \mathrm{~mm}^{2}\right)$ and their improved collection efficiencies [2] that these benefits are truly beginning to benefit those analysts wishing to measure highly beam sensitive materials.

On any EDS system, to achieve a 10 times count rate improvement requires either a 10 times increase in beam current, or a 10 times increase in solid angle. To achieve the maximum speed on a stable inorganic sample, a large increase in beam current is acceptable, however, for applications where small probe sizes and low beam densities are important to minimise sample damage and contamination, this analysis strategy is not realistic - the only real option is to maximise solid angle and collection efficiencies. New very large area SDDs therefore offer analysts of beam sensitive materials the opportunity to gather up to 15 times the counts achieved on an old $10 \mathrm{~mm}^{2}$ detector - without changing any other collection conditions.

Here, we present comparative results from a biological sample, analysed with both a traditional $10 \mathrm{~mm}^{2}$ EDS detector and a single Oxford Instruments X-Max ${ }^{N} 150 \mathrm{~mm}^{2}$ SDD detector at $4 \mathrm{kV}$ on a TESCAN Mira FEGSEM. Figure 1 shows a wheat seed which has been embedded into a Spurr type resin and stained with $\mathrm{OsO}_{4}$ to highlight the fatty acids in the oily bodies surrounding the phospho-rich structures in the centre of the aleurone cells. The aleurone cell layer was then analysed with EDS. The Os and P peaks overlap in the EDS spectrum (Figure 2) but were deconvoluted and separated at each pixel-using AZtec 'TruMap' software to ensure well defined, accurate elemental X-Ray maps are obtained.

Figure 3 shows the TruMap results achieved with the $10 \mathrm{~mm}^{2}$ EDS detector (Figure 3a) compared against those taken with the X-Max ${ }^{\mathrm{N}} 150$ EDS detector (Figure $3 \mathrm{~b}$ ). The maps were collected at $4 \mathrm{kV}$ in a single frame with a total collection time of 3 minutes. The data collected with the X-Max ${ }^{\mathrm{N}} 150$ EDS detector is clearly superior in both counts and image definition. The oily bodies are clearly delineated in the Os map (blue) and easily identified as separate structures from the P-rich areas (in yellow).

Counting for longer using the $150 \mathrm{~mm}^{2} \mathrm{X}-\mathrm{Max}^{\mathrm{N}}$ detector (30 minutes) yields further improved signal to noise and even offers sufficient structure in the Os map to enable individual oily bodies to be identified (Figure 3c). All the maps were collected with drift correction software on meaning no sample drift despite the relatively long acquisition time. Combining both the Os and $\mathrm{P}$ maps into a single layered image (Figure 3d) clearly illustrates the differing elemental distributions found across the sample. 


\section{Conclusions:}

The introduction of new large area SDD EDS detectors offers biological analysts a new way of collecting important information about their samples. Higher collection efficiencies mean higher count rates but crucially, without any need to change the current SEM operating conditions. The result is that truly informative EDS data can now be collected on biological or beam sensitive materials without compromising the sample.

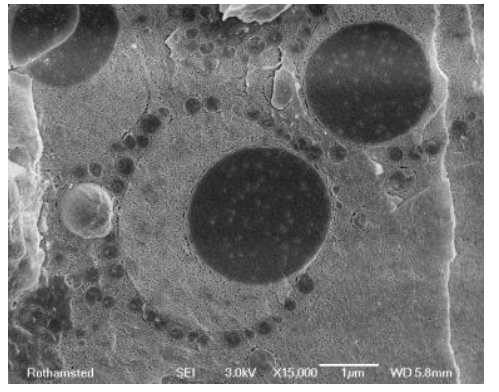

Figure 1. SEM image showing the internal structure of a wheat aleurone cell.

(a)
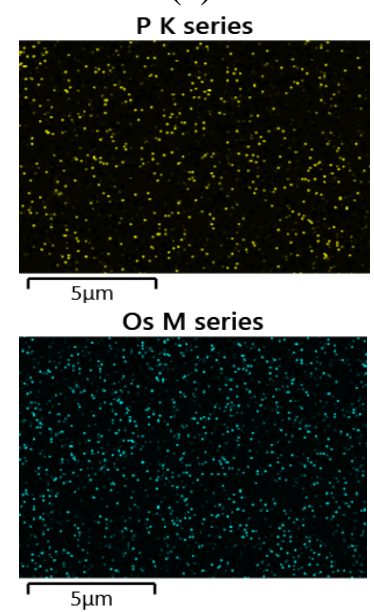

(b)
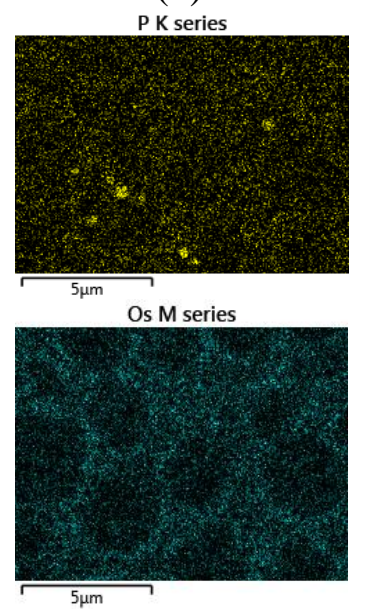

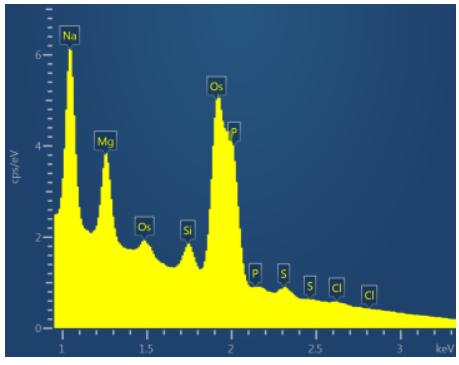

Figure 2. EDS sum spectrum of a wheat seed showing the overlap between Os \& P.

(c)

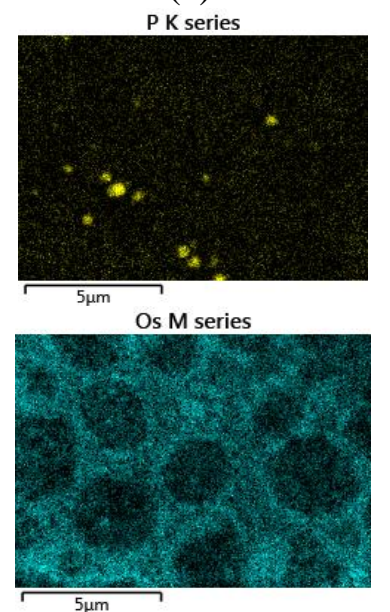

(d)

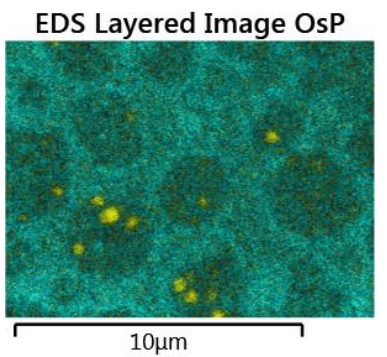

Figure 3 (a) Traditional 10mm2 EDS Detector, 3 minute maps, (b) Single X-Max ${ }^{\mathrm{N}}$ $150 \mathrm{~mm}^{2}$ EDS detector, 3minute maps (c) Single X-Max ${ }^{\mathrm{N}} 150 \mathrm{~mm}^{2}$ EDS detector, 30 minute maps (d) EDS layered image showing combined 30 minute Os and P maps.

\section{References:}

[1] "A to Z of Technology - Software for Better Results with Faster Sensors", S Burgess et al, Oxford Instruments. Microscopy \& Microanalysis 2011

[2] "High Throughput, High Quality Analysis in the Electron Microscope"; A Hyde et al, Oxford Instruments, Microscopy \& Microanalysis 2013

[3] Oxford Instruments would like to thank Jean Devonshire of Rothamsted Research, UK for providing the wheat sample for analysis as well as the SEM image seen in Figure 1. 